\title{
New Ni-Anthracene Complex for Selective and Sensitive Detection of 2,4,6-Trinitrophenol
}

\author{
Kumbam Lingeshwar Reddy, Anabathula Manoj Kumar, \\ Abhimanew Dhir $(\mathbb{D}$, and Venkata Krishnan
}

School of Basic Sciences and Advanced Materials Research Center, Indian Institute of Technology Mandi, Mandi, Himachal Pradesh 175005, India

Correspondence should be addressed to Abhimanew Dhir; abhimanew@iitmandi.ac.in and Venkata Krishnan; vkn@iitmandi.ac.in

Received 26 August 2017; Accepted 4 January 2018; Published 6 February 2018

Academic Editor: Guillermo Moyna

Copyright (C) 2018 Kumbam Lingeshwar Reddy et al. This is an open access article distributed under the Creative Commons Attribution License, which permits unrestricted use, distribution, and reproduction in any medium, provided the original work is properly cited.

\begin{abstract}
Selective and sensitive detection of explosive materials through a simple approach is an attractive area of research having implications on public safety and homeland security. Considering this implication in mind, a new Ni-anthracene complex was designed and synthesized and has been demonstrated as an efficient fluorescence chemosensor for the selective and sensitive detection of 2,4,6-trinitrophenol. Firstly, a fluorescent anthracene ligand (A) was synthesized by treating anthracene9-carboxaldehyde with 1,3-diaminopropane in presence of a weak acid. To achieve superior selectivity and great quenching efficiency for 2,4,6-trinitrophenol (TNP), a Ni complex, namely, $\left[\mathrm{Ni}\left(\mu_{2}-\mathrm{L}\right)\left(\mathrm{NO}_{3}\right)\right](\mathbf{B})$, was synthesized via the reaction of $\mathbf{A}$ with $\mathrm{Ni}\left(\mathrm{NO}_{3}\right)_{2} \cdot 6 \mathrm{H}_{2} \mathrm{O}$. Complex B showed strong emission peak $\left(\lambda_{\max }\right)$ at $412 \mathrm{~nm}$ and exhibited high selectivity towards TNP among other nitroaromatics and anions. 100 equivalents of TNP made 95\% fluorescence quenching of $\mathbf{B}$ and its detection limit for TNP was calculated as $2.8 \mu \mathrm{M}$.
\end{abstract}

\section{Introduction}

Nitroaromatic compounds are generally used in many industries for the preparation of dyes, pharmaceuticals, rubber products, explosives, chemical fibres and pesticides, and so forth [1]. These nitroaromatic compounds were released into the environment as industrial wastage and are one of the main reasons for the pollution. Moreover, due to their poisonousness, carcinogenicity to living beings, and the risk to homeland safety, they cause serious intimidations to our lives [2] . 2,4,6-TNT; 2,4-DNT; 2,4-DNB; 2,6-DNT; and 2,4,6TNP are the key constituents of environmental pollutants and explosive products [3]. Out of these compounds, TNP in particular creates health hazards and chronic, skin, and eye diseases and is used as a powerful explosive similar to TNT [4]. Furthermore, TNP is a more potent explosive than the more commonly used explosive TNT [5].

Various detection techniques such as electrochemical sensing, cyclic voltammetry, Raman spectroscopy, Liquid chromatography-mass spectrometry (LC-MS), photoluminescence spectroscopy (PL), and several other techniques have been used to detect different types of explosives including TNP. Nevertheless, most of them are pretty sophisticated, time taking, and/or difficult to operate [6,7]. Among these reported procedures, PL based method offers shorter response time and improved sensitivity and is economically worthwhile $[7,8]$. Hence, with the above stated problems from TNP and the benefits of PL, TNP sensing through optical detection is highly needed.

Till now, many fluorescent sensors have been developed for the detection of nitroaromatic explosives [7-10]. Nitroaromatics are electron deficient in nature; due to this reason some electron-rich fluorescent probes can always produce nonfluorescent Meisenheimer or $\pi$-stacking complexes with nitroaromatics $[11,12]$. Selective detection of TNP is very difficult as it has strong electron affinity [13]. Through donoracceptor electron transfer mechanism, TNP can quench the fluorescence emission of various molecules such as metal 


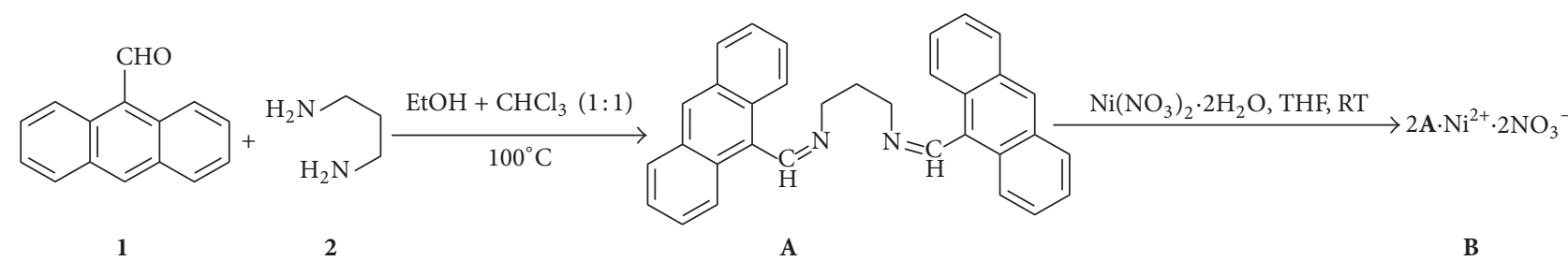

Scheme 1: Synthetic route of nickel complex B.

complexes, conjugated polymers, organic molecules, and metal-organic frameworks [14-17].

Among various fluorophores used for designing of chemosensors, anthracene based compounds have been used for the detection of several analytes, due to their high chemical stability, richness of $\pi$-electrons and strong fluorescence $[18,19]$. Mainly anthracene based compounds are widely used for the sensing of anions and cations. Nevertheless, there are very limited compounds reported for the detection of explosives/nitroaromatics [20-22]. In consequence, by considering the significance of the new photoluminescent material for TNP sensing, in this work, we synthesized a new anthracene based nickel complex with fluorescent activity for the detection of TNP. Solution phase spectral studies confirm the sensitivity of complex with TNP.

\section{Materials and Methods}

2.1. Materials. Anthracene-9-carboxaldehyde, 1,3-diaminopropane, nickel nitrate, tetra butyl ammonium salts of anions, Nitroaromatic compounds and other chemicals are purchased from Sigma-Aldrich, India and used without further purification.

2.2. Synthesis of $\boldsymbol{A}$. Anthracene-9-carboxaldehyde (0.2 g, $0.97 \mathrm{mM}$ ) was dissolved in $10 \mathrm{~mL}$ of methanol. 1,3-Diaminopropane $(0.036 \mathrm{~g}, 0.485 \mathrm{mM})$ and acetic acid $(27.8 \mu \mathrm{L}$, $0.485 \mathrm{mM}$ ) are added to the above solution while stirring. The yellow colored solution mixture was refluxed for $12 \mathrm{~h}$. Upon cooling the yellow precipitate, (7E,19E)-N-((anthracen-10$y l)$ methylene)- $N^{\prime}-(($ anthracen-9-yl)methylene)propane-1,3diamine, $\mathbf{A}$, was separated by filtration under reduced pressure and then washed with excess methanol. The product was recrystallized from methanol. Yield: $85.32 \% .{ }^{1} \mathrm{H}$ NMR $\left(500 \mathrm{MHz}, \mathrm{CDCl}_{3}, \mathrm{Me}_{4} \mathrm{Si}\right) \delta 2.57-2.54(\mathrm{t}, 2 \mathrm{H}) ; \delta 4.23-4.21$ $(\mathrm{m}, 4 \mathrm{H}) ; \delta 7.51-7.48(\mathrm{~m}, 8 \mathrm{H}) ; \delta 8.04-8.02(\mathrm{~d}, 4 \mathrm{H}) ; \delta 8.51$ (s, $2 \mathrm{H}) ; \delta 8.58-8.56(\mathrm{~d}, 4 \mathrm{H}) ; \delta 9.56(\mathrm{~s}, 2 \mathrm{H})$ (see supplementary Figure $\mathrm{S} 1) ;{ }^{13} \mathrm{C}$ NMR $\left(\mathrm{CDCl}_{3}\right): \delta 160.74,131.32,130.05$, $129.31,128.88,128.24,126.71,125.25,124.80,60.89,32.48$ (see supplementary Figure S2); HR-MS Data: $[\mathrm{M}+1]^{+}=450.86$.

2.3. Synthesis of $\boldsymbol{B}$. To the solution of $\mathbf{A}(100 \mathrm{mg}, 0.22 \mathrm{mM}$ in THF), nickel nitrate $\left[\mathrm{Ni}\left(\mathrm{NO}_{3}\right)_{2} \cdot 6 \mathrm{H}_{2} \mathrm{O}, 0.56 \mathrm{~g}, 1.93 \mathrm{mM}\right]$ was added and stirred for two hours at room temperature. The yellowish green precipitate was separated out under reduced pressure. Yield: $83.96 \%$. HR-MS $[\mathrm{M}+1]^{+}$found 1082.34 .

2.4. Characterizations. The ${ }^{1} \mathrm{H}$ and ${ }^{13} \mathrm{C}$ NMR spectra of the A have been recorded on a Bruker $400 \mathrm{MHz}$ NMR instrument. Mass spectra of the $\mathbf{B}$ have been recorded on Kratos PC Axima HR-Mass spectrometer in linear mode. The fluorescence quenching measurements were performed using Cary Eclipse Fluorescence spectrophotometer. The excitation and emission slit widths (each $10 \mathrm{~nm}$ ) and scan rate $\left(500 \mathrm{~nm} \mathrm{~min}{ }^{-1}\right)$ were kept constant for all the measurements. The optical absorption spectral measurements were recorded using a Shimadzu UV-2450 spectrophotometer with a quartz cuvette (path length, $1 \mathrm{~cm}$ ). Energy dispersive X-ray spectra (EDAX) were obtained using scanning electron microscope (SEM), FEI Nova Nano SEM-450.

\section{Results and Discussion}

Condensation of 1,3-diaminoprone 2 with anthracene-9carboxaldehyde $\mathbf{1}$ produced a fluorescent ligand $\mathbf{A}$, which gave a nickel complex $\mathbf{B}$ with the addition of $\mathrm{Ni}\left(\mathrm{NO}_{3}\right)_{2} \cdot 6 \mathrm{H}_{2} \mathrm{O}$ at RT (Scheme 1). The as-synthesized complex was characterized by mass spectrometry, where the parent ion $(\mathrm{m} / z)$ peak appeared at 1082 corresponding to the species $\left[2 \mathrm{~A} \cdot \mathrm{Ni}\left(\mathrm{NO}_{3}\right)_{2}\right.$ ] (see supplementary Figure S3). The presence of all the elements in complex B is confirmed through elemental analysis (see supplementary Figure S4). As we are developing a chemosensor for TNP, the main motivation behind the synthesis of compound $\mathbf{A}$ is that it was expected to become a nonfluorescent moiety as photoinduced electron transfer (PET) process can take place due to presence of imino nitrogen atom [23]. The binding of nickel ions to the imino nitrogen of compound $\mathbf{A}$ will prevent the PET process and it eventually results in the perseverance of fluorescence. Consequently, TNP can perhaps quench the fluorescence emission of $\mathbf{B}$ with electron transfer or energy transfer mechanism and could be sensed [24].

Complex B $(1 \mu \mathrm{M})$ showed reasonably good emission intensity of anthracene monomeric species at $412 \mathrm{~nm}$ in THF : HEPES (9.5:0.5) when excited at $\lambda_{\text {ex }}=365 \mathrm{~nm}$ [25]. On addition of TNP $(150 \mu \mathrm{M})$ to the solution of $\mathbf{B}(1 \mu \mathrm{M})$ in THF : HEPES $(9.5: 0.5)$, the emission band shows quenching of emission band (Figure 1). To observe the selectivity of $\mathbf{B}$ towards TNP, we carried out the fluorescence titration experiments with similar amount of $150 \mu \mathrm{M}$ other nitroderivatives, namely, nitrotoluene (NT), 2,4-dinitrotoluene (2,4 DNT), and 1,4-dinitrobenzene $(1,4 \mathrm{DNB})$ and anions $\left(\mathrm{F}^{-}, \mathrm{Cl}^{-}, \mathrm{Br}^{-}\right.$, $\mathrm{I}^{-}, \mathrm{OH}^{-}, \mathrm{HSO}_{3}{ }^{-}, \mathrm{CN}^{-}, \mathrm{NO}_{3}{ }^{-}, \mathrm{CH}_{3} \mathrm{COO}^{-}, \mathrm{ClO}_{4}{ }^{-}$, and $\mathrm{PO}_{4}{ }^{-}$) with tetrabutyl ammonium as counter ion, and observed no significant change in the emission spectrum of $\mathbf{B}$ depicting the selectivity of $\mathbf{B}$ (Figure 2). Detection limit (DL) was calculated using the equation: $\mathrm{DL}=3 \alpha / K$, where $\alpha$ is the 


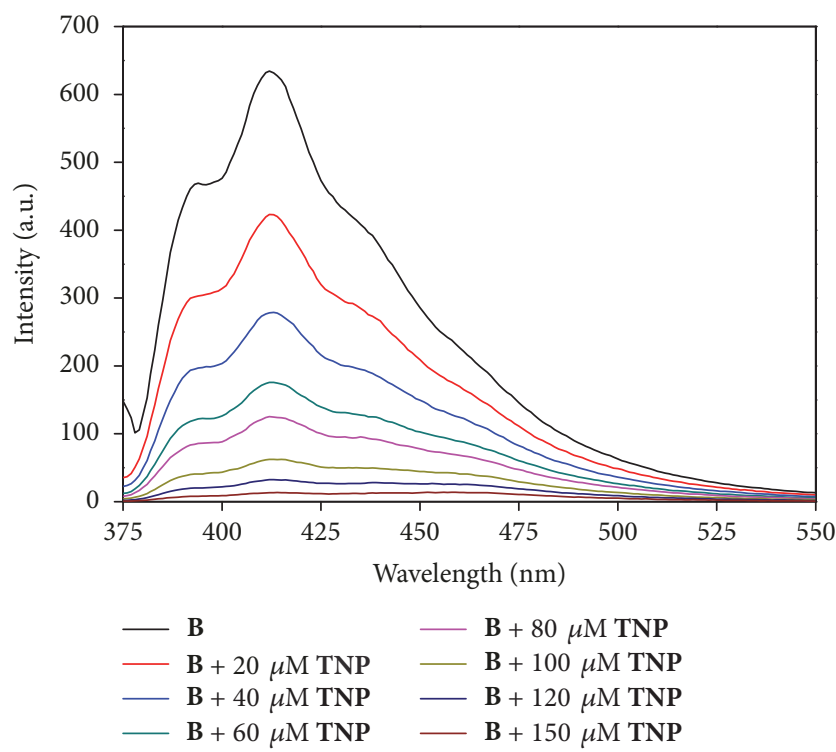

FIGURE 1: Change in emission spectrum of $\mathbf{B}(1 \mu \mathrm{M})$ on addition of TNP $(150 \mu \mathrm{M})$ in THF : HEPES $(9.5: 0.5)(\nu / \nu)$.

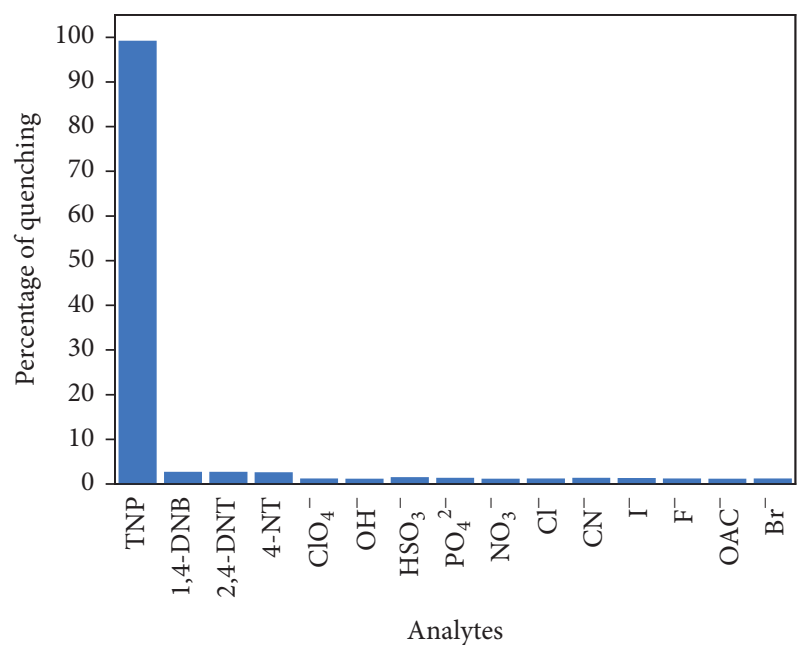

FIGURE 2: Fluorescence response of $\mathbf{B}(1 \mu \mathrm{M})$ in THF: HEPES $(9.5: 0.5)(\nu / \nu)$ among various nitroaromatics and anions. All analytes are tested in the same molar ratio to $\mathbf{B}$.

standard deviation calculated by measuring the fluorescence intensity of complex $\mathbf{B}$ for more than 15 times and $K$ presents the slope of fitting curve between variation of the fluorescence intensity $\left(I_{0}-I\right)$ and the added amount of TNP at low concentration. According to the linear fitting curve (Figure S5 shown in supporting information), the detection limit of complex B for sensing of TNP was found to be $2.857 \mu \mathrm{M}$ [26]. The complex B DL in sensing of TNP is as comparable with the existing materials (see Table S1 in supporting information).

We also examined the meddling of other anions and nitroaromatics towards the sensing of TNP (Figure 3 ) and it was confirmed that the $\mathbf{B}$ was detecting TNP even in the presence of other potential analytes. The emission plot of $\mathbf{B}$

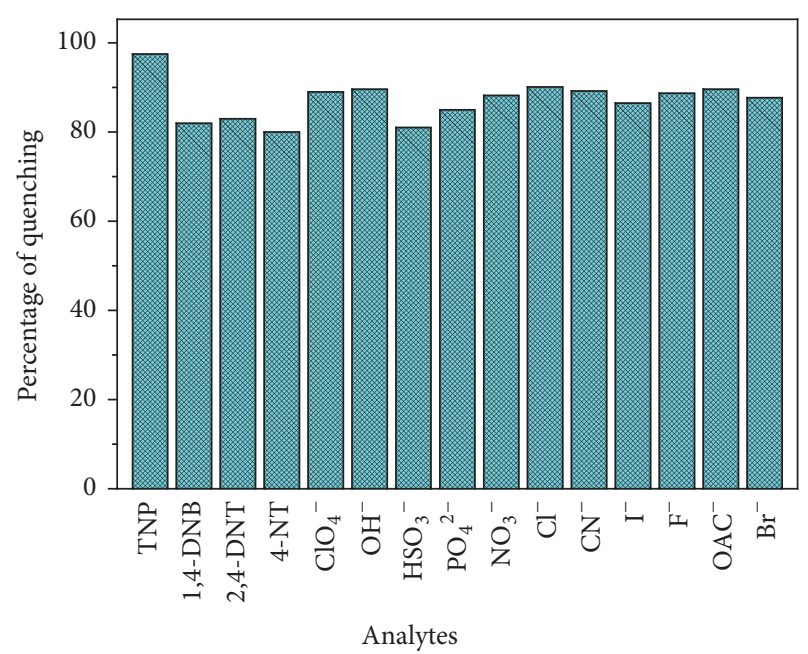

FIGURE 3: Fluorescence quenching in $\mathbf{B}$ with the addition of TNP alone and TNP in the presence of other analytes. The molar ratio to B used in each case is 100 equivalents.

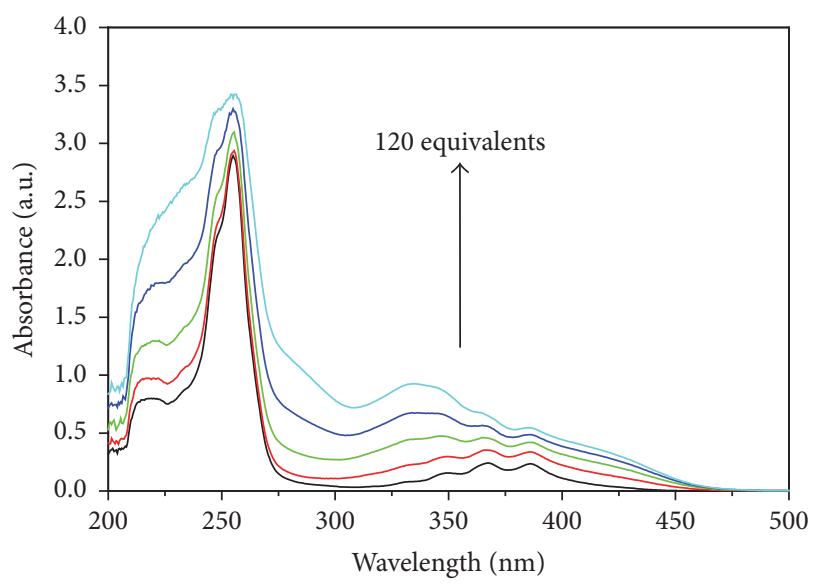

FIgURE 4: Change in absorption spectra of $4(1 \mu \mathrm{M})$ with the addition of TNP in a mixed aqueous medium of THF: HEPES $(9.5: 0.5)(\nu / \nu)$.

in the presence of TNP was studied using Stern-Volmer relationship. Stern-Volmer plot seemed like a hyperbolic curve (see supplementary Figure S6) which may be accredited to the combination of static and dynamic (collision) quenching [27].

We have also detected these variations with the assistance of UV-vis analysis. Nickel complex $\mathbf{B}(1 \mu \mathrm{M})$ in THF : HEPES (9.5: 0.5) showed an absorbance band at $260 \mathrm{~nm}$ (Figure 4). On addition of TNP $(120 \mu \mathrm{M})$ along with the formation of band at $340 \mathrm{~nm}$ corresponding to TNP (Figure 4), there was subsequent increase in the band at $260 \mathrm{~nm}$ presenting that there is some photochemical process happening between $\mathbf{B}$ and TNP. To have a deep insight into the mechanism, we made plot between the absorbance spectrum of TNP and emission spectrum of $\mathbf{B}$ (Figure 5). The overlap between the two plots suggests an energy transfer mechanism from photoexcited $\pi$-electron-rich derivative $\mathbf{B}$ to ground state 


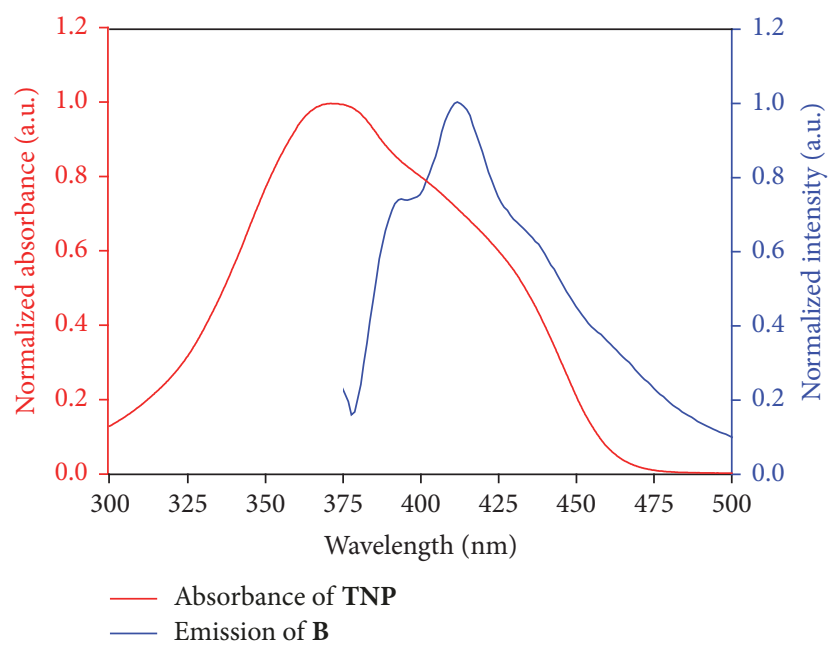

FIGURE 5: Spectral overlap of the absorption of TNP (red line) with the emission spectrum of $\mathbf{B}$ (blue line).

electron-deficient TNP. Thus, the changes occurring in the optical properties of $\mathbf{B}$ on addition of TNP are attributed to the energy transfer process.

\section{Conclusions}

In conclusion, we have designed and synthesized a new photoluminescent Ni-anthracene complex. The luminescence behaviour of the complex was assessed towards different nitroaromatics and other analytes. The synthesized $\mathrm{Ni}$ anthracene complex shows selective and sensitive detection of TNP and could be a helpful tool for the people working in forensic sciences.

\section{Conflicts of Interest}

The authors declare that they have no conflicts of interest regarding the publication of this article.

\section{Acknowledgments}

Advanced Materials Research Center (AMRC), IIT Mandi, is gratefully acknowledged for the laboratory and characterization facilities. Abhimanew Dhir and Venkata Krishnan acknowledge Department of Science and Technology, India, for INSPIRE faculty award. Kumbam Lingeshwar Reddy is grateful to Ministry of Human Resource Development (MHRD), India, for research fellowship.

\section{Supplementary Materials}

Figure S1: ${ }^{1} \mathrm{H}$ NMR spectrum of A. Figure $\mathrm{S} 2:{ }^{13} \mathrm{C}$ NMR spectrum of A. Figure S3: mass spectrum of complex B. Figure S4: energy dispersive X-ray spectrum (EDAX) of complex $\mathbf{B}$. Figure S5: change in fluorescence intensity of complex $\mathbf{B}$ at low concentrations of TNP. Figure S6: Stern-Volmer plot for the quenching of the fluorescence of $\mathbf{B}$ upon the addition of $120 \mu \mathrm{M}$ of TNP. Table S1: comparison of detection limit with different reported materials. (Supplementary Materials)

\section{References}

[1] P. Kovacic and R. Somanathan, "Nitroaromatic compounds: environmental toxicity, carcinogenicity, mutagenicity, therapy and mechanism," Journal of Applied Toxicology, vol. 34, no. 8, pp. 810-824, 2014.

[2] G. He, H. Peng, T. Liu, M. Yang, Y. Zhang, and Y. Fang, "A novel picric acid film sensor via combination of the surface enrichment effect of chitosan films and the aggregation-induced emission effect of siloles," Journal of Materials Chemistry, vol. 19, no. 39, pp. 7347-7353, 2009.

[3] S. K. Samanta, B. Bhushan, A. Chauhan, and R. K. Jain, "Chemotaxis of a Ralstonia sp. SJ98 toward different nitroaromatic compounds and their degradation," Biochemical and Biophysical Research Communications, vol. 269, no. 1, pp. 117-123, 2000.

[4] J. Shen, J. Zhang, Y. Zuo et al., "Biodegradation of 2,4,6trinitrophenol by Rhodococcus sp. isolated from a picric acidcontaminated soil," Journal of Hazardous Materials, vol. 163, no. 2-3, pp. 1199-1206, 2009.

[5] M. E. Germain and M. J. Knapp, "Optical explosives detection: from color changes to fluorescence turn-on," Chemical Society Reviews, vol. 38, no. 9, pp. 2543-2555, 2009.

[6] S. Chen, Q. Zhang, J. Zhang, J. Gu, and L. Zhang, "Synthesis of two conjugated polymers as TNT chemosensor materials," Sensors and Actuators B: Chemical, vol. 149, no. 1, pp. 155-160, 2010.

[7] M. S. Meaney and V. L. McGuffin, "Luminescence-based methods for sensing and detection of explosives," Analytical and Bioanalytical Chemistry, vol. 391, no. 7, pp. 2557-2576, 2008.

[8] S. J. Toal and W. C. Trogler, "Polymer sensors for nitroaromatic explosives detection," Journal of Materials Chemistry, vol. 16, no. 28, pp. 2871-2883, 2006.

[9] N. Venkatramaiah, D. M. G. C. Rocha, P. Srikanth, F. A. Almeida Paz, and J. P. C. Tomé, "Synthesis and photophysical characterization of dimethylamine-derived $\mathrm{Zn}$ (II)phthalocyanines: exploring their potential as selective chemosensors for trinitrophenol," Journal of Materials Chemistry C, vol. 3, no. 5, pp. 10561067, 2015.

[10] J.-F. Xiong, J.-X. Li, G.-Z. Mo et al., "Benzimidazole derivatives: Selective fluorescent chemosensors for the picogram detection of picric acid," The Journal of Organic Chemistry, vol. 79, no. 23, pp. 11619-11630, 2014.

[11] B. Xu, X. Wu, H. Li, H. Tong, and L. Wang, "Selective detection of TNT and picric acid by conjugated polymer film sensors with donor-acceptor architecture," Macromolecules , vol. 44, no. 13, pp. 5089-5092, 2011.

[12] S. Xu, H. Lu, J. Li et al., "Dummy molecularly imprinted polymers-capped CdTe quantum dots for the fluorescent sensing of 2,4,6-trinitrotoluene," ACS Applied Materials \& Interfaces, vol. 5, no. 16, pp. 8146-8154, 2013.

[13] S. S. Nagarkar, B. Joarder, A. K. Chaudhari, S. Mukherjee, and S. K. Ghosh, "Highly selective detection of nitro explosives by a luminescent metal-organic framework," Angewandte Chemie International Edition, vol. 52, no. 10, pp. 2881-2885, 2013.

[14] V. Bhalla, S. Kaur, V. Vij, and M. Kumar, "Mercury-modulated supramolecular assembly of a hexaphenylbenzene derivative for selective detection of picric acid," Inorganic Chemistry, vol. 52, no. 9, pp. 4860-4865, 2013.

[15] W. Wu, S. Ye, L. Huang et al., "A conjugated hyperbranched polymer constructed from carbazole and tetraphenylethylene moieties: convenient synthesis through one-pot "A2 + B4" 
suzuki polymerization, aggregation-induced enhanced emission, and application as explosive chemosensors and PLEDs," Journal of Materials Chemistry, vol. 22, no. 13, pp. 6374-6382, 2012.

[16] X.-G. Hou, Y. Wu, H.-T. Cao et al., "A cationic iridium(iii) complex with aggregation-induced emission (AIE) properties for highly selective detection of explosives," Chemical Communications, vol. 50, no. 45, pp. 6031-6034, 2014.

[17] D. K. Singha, S. Bhattacharya, P. Majee, S. K. Mondal, M. Kumar, and P. Mahata, "Optical detection of submicromolar levels of nitro explosives by a submicron sized metal-organic phosphor material," Journal of Materials Chemistry A, vol. 2, no. 48, pp. 20908-20915, 2014.

[18] R. Pandey, L. Reddy, S. Ishihara, A. Dhir, and V. Krishnan, "Conformation induced discrimination between picric acid and nitro derivatives/anions with a $\mathrm{Cu}$-pyrene array: the first decision making photonic device," RSC Advances, vol. 3, no. 44, pp. 21365-21368, 2013.

[19] K. L. Reddy, A. M. Kumar, A. Dhir, and V. Krishnan, "Selective and sensitive fluorescent detection of picric acid by new pyrene and anthracene based copper complexes," Journal of Fluorescence, vol. 26, no. 6, pp. 2041-2046, 2016.

[20] B. Gole, S. Shanmugaraju, A. K. Bar, and P. S. Mukherjee, "Supramolecular polymer for explosives sensing: role of $\mathrm{H}$ bonding in enhancement of sensitivity in the solid state," Chemical Communications, vol. 47, no. 36, pp. 10046-10048, 2011.

[21] S. Shanmugaraju, S. A. Joshi, and P. S. Mukherjee, "Fluorescence and visual sensing of nitroaromatic explosives using electron rich discrete fluorophores," Journal of Materials Chemistry, vol. 21, no. 25, pp. 9130-9138, 2011.

[22] S. Shaligram, P. P. Wadgaonkar, and U. K. Kharul, "Fluorescent polymeric ionic liquids for the detection of nitroaromatic explosives," Journal of Materials Chemistry A, vol. 2, no. 34, pp. 13983-13989, 2014.

[23] B. Valeur and I. Leray, "Design principles of fluorescent molecular sensors for cation recognition," Coordination Chemistry Reviews, vol. 205, no. 1, pp. 3-40, 2000.

[24] S. R. Wallenborg and C. G. Bailey, "Separation and detection of explosives on a microchip using micellar electrokinetic chromatography and indirect laser-induced fluorescence," Analytical Chemistry, vol. 72, no. 8, pp. 1872-1878, 2000.

[25] J. N. Demas and G. A. Crosby, "The measurement of photolumineseence quantum yields. a review," The Journal of Physical Chemistry C, vol. 75, no. 8, pp. 991-1024, 1971.

[26] G. L. Long and J. D. Winefordner, "Limit of detection: a closer look at the IUPAC definition," Analytical Chemistry, vol. 55, no. 7, pp. 712-724, 1983.

[27] D. Zhao and T. M. Swager, "Sensory responses in solution vs solid state: a fluorescence quenching study of poly(iptycenebutadiynylene)s," Macromolecules, vol. 38, no. 22, pp. 9377-9384, 2005. 

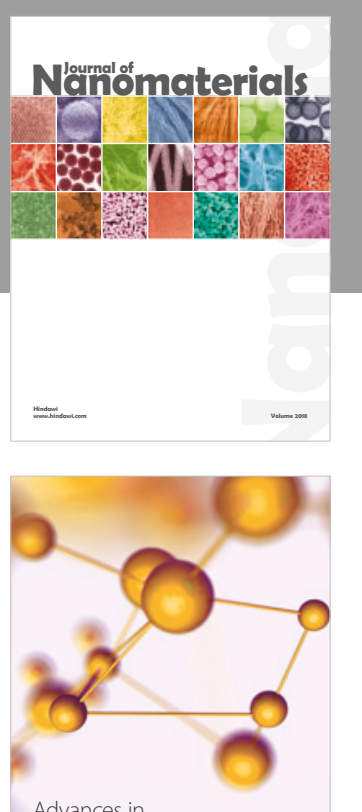

Physical Chemistry
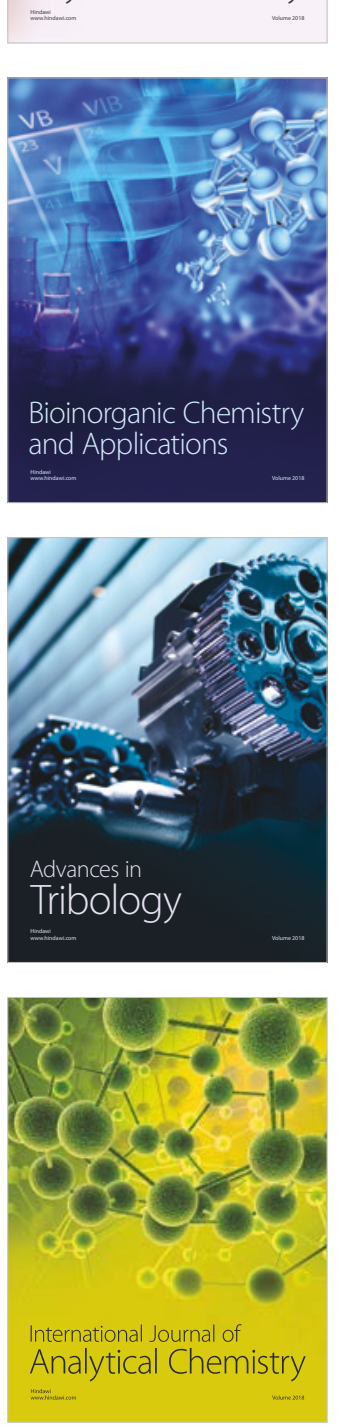

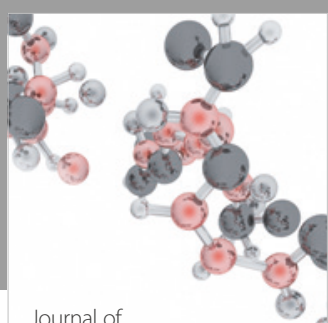

Analytical Methods

in Chemistry

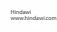

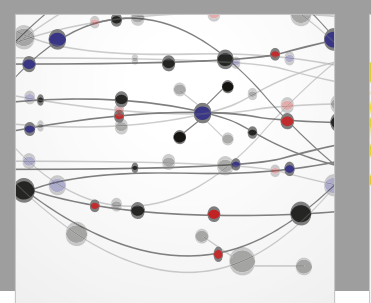

The Scientific World Journal

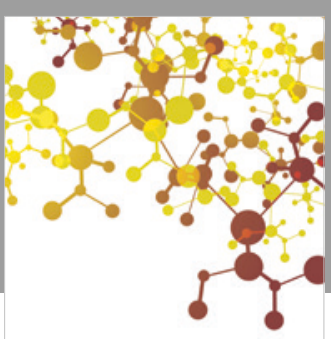

Journal of

Applied Chemistry
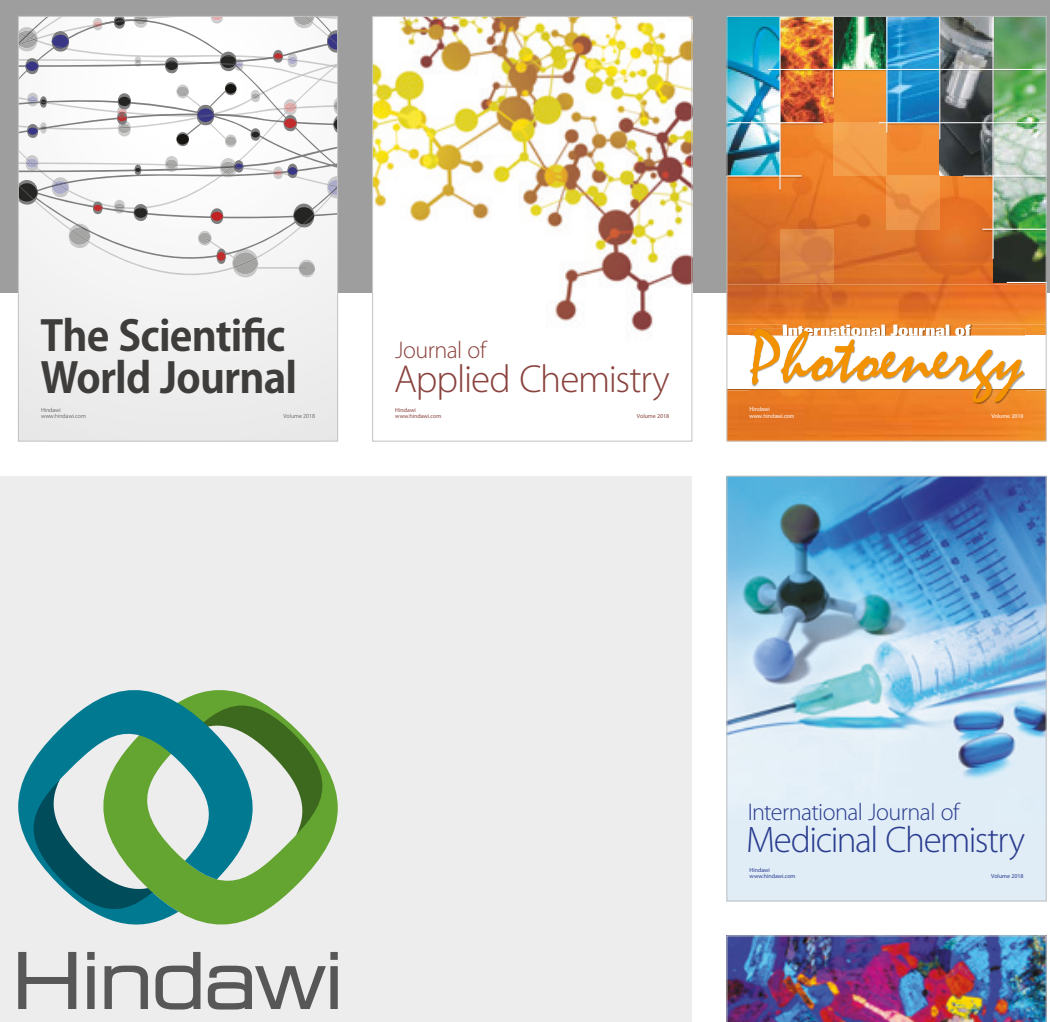

Submit your manuscripts at

www.hindawi.com
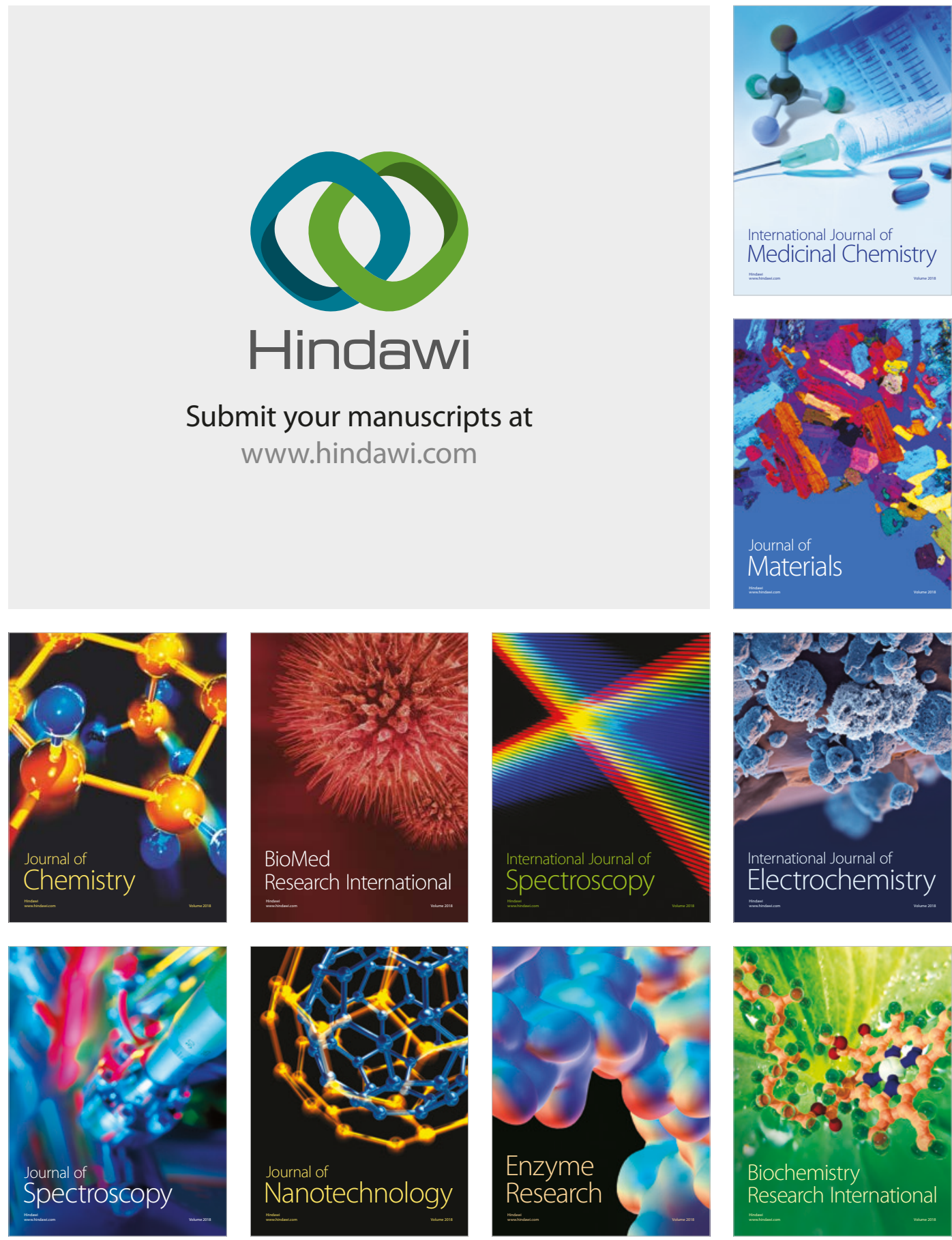
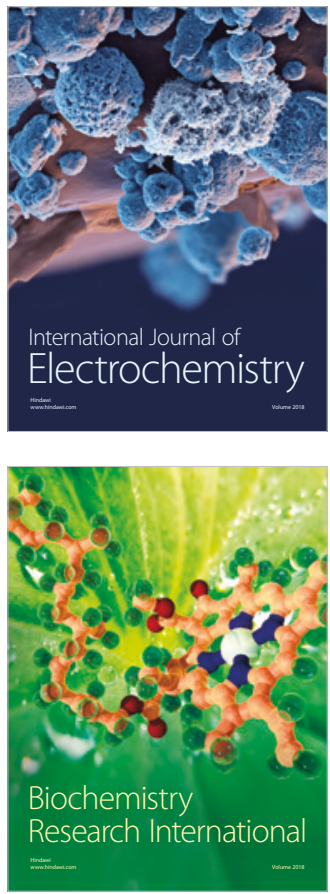